\title{
EL NUEVO MODELO INDUSTRIAL EN EL SECTOR TEXTIL DE LA PROVINCIA DE ALICANTE (1970-1991)*
}

\author{
Rafael Sebastiá Alcaraz
}

\section{RESUMEN}

Los espacios industriales vertebrados alrededor de la industria textil en la provincia de Alicante, han atravesado una profunda crisis desde 1970 hasta 1991. Las dificultades mostradas para adaptarse a las innovaciones, a la apertura de los mercados y al incremento de la competencia de países que disponen de abundante materia prima o de mano de obra más barata han modificado sus características geográficas.

Palabras claves: Alicante, localización industrial, textil.

\begin{abstract}
The industrial areas vertebrated around the textile industry in the province of Alicante, have gone through deep crisis from 1970 until 1991. The difficulties shown to adapt to the novelties, the opening of the markets and an increasing competence of the countries with lots of raw material an with a cheaper labourforce have modified its geographical characteristics.
\end{abstract}

Key words: Alicante, industrial location, textile.

\section{Introducción}

El sector textil fue pionero en el tránsito de la manufactura a la industria. Estudios como el de Ismael Vallés ${ }^{1}$ y Vicente Gozálvez ${ }^{2}$ explican la mecanización y la consolida-

* Investigación realizada dentro del Proyecto G.V. 2401/94.

1 VALLÉS, Ismael: Industria tèxtil i societat a la Regió Alcoi-Ontinyent 1780-1930, Institut Valencià d'Estudis i Investigació, Universitat de Valencia, 1986, 197 pp.

2 GOZÁLVEZ PÉREZ, Vicente: La industria de esteras y alfombras en Crevillente, Alicante, Círculo de Economía, 1975, 41 pp. + 8 lám. f. t. 
ción de la industria textil alicantina en la época contemporánea. Otros trabajos, como el de R. Ma. Jordá3 o el de Juan $\mathrm{Such}^{4}$, analizan cual es la situación de esta industria.

La industria textil nos ofrece una gran variedad de clasificaciones y subsectores: atendiendo a las materias primas que se utilizan, se encuentran especialidades como las de la lana, cáñamo y yute, algodón, seda y fibras sintéticas; desde el punto de vista estadístico se clasifican en preparación, hilatura y tejidos, acabados, y alfombra. La producción del sector textil también ofrece una gran heterogeneidad, abarcando a los tejidos especiales, de hogar, alfombras, etc. Las actividades incluidas ofrecen tanto productos finales (géneros de punto o alfombras) como intermedios (hilos y telas).

El modelo de localización de la industria textil se podría aproximar al definido como multipolar por J. Luis Cevidades y Gaspar Hernández 5 . La industria textil se localiza, según este modelo, de forma concentrada sobre una serie de espacios, cada uno de ellos especializado en un subsector. Uno de estos espacios se localiza alrededor de Crevillent, cuya actividad textil predominante es la de la alfombra. Otro se sitúa al norte de la provincia de Alicante y sur de Valencia, estructurado sobre dos municipios, Alcoi y Ontinyent. La zona de Alcoi se centra en los textiles para el hogar y junto con los municipios vecinos de Banyeres, Cocentaina y Muro concentra la mayor parte de los acabados textiles de la provincia. Un tercer grupo está disperso y a su vez integrado por municipios especializados en una sola actividad textil, pero sin un ciclo completo de producción.

Estos centros o cabeceras cuentan con una gran tradición textil y aunque funcionan de forma independiente, con frecuencia se establecen contactos, entre otros, de tipo técnico o de formación; basta recordar la influencia de la Escuela Técnica Industrial de Alcoi (actualmente EPSA) o la de AITEX.

La evolución de la industria textil provincial se inscribe en un espacio más amplio, cuyo punto de referencia es la Comunidad Valenciana, España y la CEE. La crisis textil, que se inicia en 1960, estaba anunciada desde la década anterior, cuando se incorporaron nuevos países, algunos de ellos recién descolonizados, a esta actividad industrial. Los bajos costes laborales fueron los que permitieron la incorporación a los mercados mundiales, de modo que los países europeos fueron perdiendo cuotas de mercado. Las grandes empresas constructoras de maquinaria textil distribuyeron la tecnología necesaria entre estos países en vías de industrialización; a los países europeos no les quedó otra solución que buscar otras vías alternativas según la estructura productiva dominante en cada región. La reconversión se hizo necesaria y las medidas que se adoptaron fueron múltiples. En Gran Bretaña, cuna de la revolución industrial, las grandes empresas tendieron a integrar las fases productivas. En Alemania las empresas medianas procedieron a la especialización y modernización de sus equipos productivos. En Italia las pequeñas empresas se agruparon en Distritos Industriales buscando una flexibilidad que les permitiera adaptarse a los rápidos cambios del entorno 6 .

3 JORDÁ BORRELL, R.M.: «Alcoy, la crisis textil de 1965 y sus repercusiones» Cuadernos de Geografía, ${ }^{\circ} 18$, Universidad de Valencia, 1977, pp. 27-35.

4 SUCH JUAN, Juan: La industria textil de Alcoi-Ontinyent, Gráficas Ciudad, Alcoy, 1993, 302 pp.

5 CIVIDADES HERNÁNDEZ, J. Luis; HERNÁNDEZ HERNÁNDEZ, Gaspar: «Dinámica industrial y áreas de especialización», Estructura Económica de la Provincia de Alicante, Diputación Provincial de Alicante, 1993, pp. 173-188.

6 BOUSSEMART, Benôit; DE BANDT, Jacques: «The european textile industries: Widely y varying structures» en H.W. De Jong (ed.): The structure of European industry, Kluwer Academic Publishers, 1988, pp. 183-209. 
En la provincia de Alicante el cambio en la industrial textil desde 1970 a 1991 coincide con un cambio general en el sector secundario. De este modo, se pasa de un modelo extensivo cuyos rasgos más representativos han sido:

1.- Predominio de la pequeña y mediana empresas, como consecuencia de la evolución de los talleres artesanales.

2.- Industria de bienes de consumo final.

3.- Empleo de mano de obra abundante y barata.

4.- Utilización de equipamientos y tecnologías sencillos.

5.- Bajos costes de inversión como resultado del apartado anterior.

6.- Aprovechamiento inicialmente de materias primas locales, aunque en estas fechas en su mayoría eran importadas.

7.- Desarrollo del comercio exterior como resultado de las diferencias en la producción de los costes salariales.

A otro nuevo modelo industrial que queda definido por las siguientes características:

1.- Predominio de la pequeña y mediana empresas, favorecida por la difusión de la técnica.

2.- Las industrias continúan fabricando mayoritariamente bienes de consumo final, calzado, alimentación, textil, juguete, etc.

3.- Sustitución de la mano de obra compensada por una mejora de los equipamientos industriales, como señala la mayor potencia instalada por puesto de trabajo previsto crear.

4.- Intensificación de la inversión por inscripción.

5.- Mayor inversión, tanto por puesto de trabajo previsto crear, como por inscripción, fruto de un proceso donde se sustituye la mano de obra por capital.

6.- Esta transformación se percibe mejor en las industrias que amplían que en las de nueva creación.

7.- Dependencia de las materias primas, y ahora también de las tecnologías importadas.

8.- Ampliación de los mercados con la integración europea e incremento de las relaciones con este espacio. En el comercio exterior alicantino la competitividad se consigue a través de las innovaciones.

\section{La localización geográfica}

La industria textil valenciana, al final del período estudiado, 1991, había aumentado su importancia dentro de España. En la Comunidad Valenciana esta industria se localiza en su mayoría alrededor de la carretera nacional 340, que atraviesa de Norte a Sur dicho espacio, y en sus radiales. Además, se asiste a una especialización por comarcas como son, textiles para el hogar en Alcoi, Cocentaina, Muro, Albaida y Banyeres; mantas en Ontinyent, Bocairent, Agullent; alfombra y redes en Crevillent, Callosa del Segura; género de punto en Alcoi ${ }^{7}$, y cada vez más en Muro.

Durante 1970, en la provincia de Alicante se situaban más de la mitad (54’3\%) de las empresas textiles de la Comunidad Valenciana y sólo el 40'5\% de los empleos. Este sector provincial representaba, a su vez, el 11'8\% de las empresas del total nacional y el 5'3\% del empleo, datos que destacan el menor número de trabajadores por empresa.

7 En 1980 la producción de Género de Punto se concentraba en la provincia de Valencia (57\%), Alicante (25\%) y el resto en la de Castellón. En las capitales de provincia se localizaba el 28\% y en Alcoy el 13\% según el Libro Blanco sobre las repercusiones en la economía valenciana de la adhesión de España a las Comunidades Europeas, publicado por la Consellería de Economía y Hacienda. 
Según el censo de 1978, dentro de la provincia, las empresas textiles se concentraban en Alcoi y en municipios inferiores a 20.000 habitantes, que representaban la mitad de las existentes en la provincia. En el Libro blanco se señala, además, que en 1980 el 93\% de todas las empresas españolas de la alfombra se localizaban en Crevillent, localidad que producía el 90\% del total del subsector. La concentración se explica porque, a parte de los factores históricos, el reglamento del Plan de Reestructuración de 1973 dificultaba la instalación de empresas de alfombras tejidas en otras zonas de España. En la provincia de Alicante y en la de Valencia se observa una cierta especialización en el denominado subsector tejidos-hogar ${ }^{8}$.

La actividad textil, con datos comarcales de 1980, presenta una fuerte concentración dentro de la Comunidad Valenciana en los denominados Valls d'Alcoi, seguida por la Vall d'Albaida; una concentración bastante menor se encuentra en el Camp d'Elx y en la Vega Baja.

La industria textil provincial, analizando el directorio de empresas de 1991, conserva las características generales del período estudiado, es decir:

1.- Los principales municipios textiles se localizan en torno a Alcoi, que concentran el $22 \%$ de las empresas de la provincia, y en Crevillent (15\%). Crevillent agrupa en 1990 el 90\% de la producción de la alfombra en España; el subsector de la alfombra da empleo aproximadamente a unas 60 empresas, que ocupan cerca de 2.000 personas, produciendo en 1989 una cifra global de 16.000 millones de $\mathrm{m}^{2}$.

2.- Alrededor de Alcoi se vertebra una comarca textil que suma el 61'9\% de las empresas del sector y está integrada por Banyeres, Biar, Cocentaina, Gaianes, L'Orxa, Muro y Villena. A estos se suman, según consta en otras fuentes, Alcosser, Alfafara, Beniarrés, Benilloba, Beneixama, Camp de Mirra y Canyada.

3.- Una cierta dispersión por toda la provincia de esta actividad industrial. Entre los municipios que figuran al menos con una empresa, además de los mencionados anteriormente, se encuentran Albatera, Almoradí, Alicante, El Campello, Callosa del Segura, Elx, Orihuela y La Vila Joiosa. En algunas de estas localidades la actividad textil depende de un mercado específico. Por ejemplo, en La Vila Joiosa la actividad textil se dedica a la fabricación de redes para pesca; en este subsector Callosa del Segura concentra casi todas las empresas y Almoradí, en sus proximidades, también cuenta con una empresa.

Para el sector textil, productor de un bien de consumo que requiere frecuentemente una transformación posterior, y que constituye un input para actividades como la del juguete, calzado, mueble, etc., se puede considerar que los costes de transporte no afectan de forma decisiva a su localización. En otros tiempos, el aprovechamiento de la energía hidráulica pudo influir de forma importante en su localización, no obstante en la actualidad tiende a ubicarse en zonas bien comunicadas y abastecidas de energía fácilmente. Sin embargo, la tradición y el conocimiento de los procesos productivos han sido bastante decisivos en el momento de localizar las industrias. La saturación de los núcleos vertebradores, la falta de infraestructuras y las deseconomías influyeron en la dispersión de esta industria. Un ejemplo paradigmático es el caso de la industria textil de Alcoi. Los efectos de los movimientos centrífugos de Alcoi se hallan estudiados desde el punto de vista del receptor en el caso del municipio de Muro $^{9}$, donde se aprecia que la existencia de suelos baratos, bien comunicados, con buen abastecimiento de agua y mano de obra barata y con una cierta tradición industrial, fueron elementos decisivos para el asentamiento de industrias

8 COCIN de España, Informe Peat Marwick, 20 Volúmenes, Noviembre 1992.

9 SEBASTIÀ ALCARAZ, Rafael: «El proceso de industrialización de Muro», Ayudas a la investigación, Vol. VI, Ed. Instituto de Cultura Juan-Gil Albert, Alicante, 1993, pp. 143-151. 
textiles alcoyanas. Las consecuencias fueron que a las tradicionales industrias de transformados agrícolas y textiles se sumaron otras alóctonas, que modificaron definitivamente la estructura económica del municipio, produciendo importantes alteraciones en la morfología urbana. En este municipio el planeamiento también fue posterior a la necesidad de suelo industrial; sin embargo, las industrias se asentaron sobre unos espacios más o menos definidos, próximos a carreteras y a la estación del ferrocarril.

La «globalización» de la economía mundial influyó de forma muy importante en la localización de la industria textil. En primer lugar, como se ha afirmado anteriormente, la localización de esta actividad no se halla en todas las fases del proceso productivo «determinada» por el precio del coste de los transportes; en segundo lugar, la difusión de la técnica y la elevada incidencia del precio de la mano de obra han favorecido su dispersión por los países en vías de desarrollo. Este movimiento centrífugo se inició en la industria de la confección en la década de los cincuenta, el de las actividades de tejeduría y acabados en la década de los sesenta; posteriormente, en la de los setenta se produjo el traslado de la fabricación de fibras ${ }^{10}$.

\section{Características del sector}

\section{a) El tamaño de las empresas}

Al inicio del período estudiado, 1970, las industrias textiles en la provincia de Alicante eran mayoritariamente pequeñas (52'6\%, de 1 a 10 empleados) y las grandes (más de 100 empleados) representaban un porcentaje reducido (4'1\%), sin que ninguna sobrepasara los 500 empleados (Cuadro 1).

Esta situación de mayor predominio de la pequeña empresa en la provincia de Alicante se ha comprobado, una vez pasada la crisis, que ha resultado positiva, pues frente al modelo fordista imperante en la época, las empresas pequeñas demostraron una mayor capacidad para adaptarse y superar las dificultades.

Otra característica de la actividad textil ha sido la tendencia a reducir empleo. Las dificultades del mercado y la mejora de la competitividad mediante la renovación tecnológica y dotación de infraestructura (reconversión) han contribuido a que se produzca este hecho. De esta forma, se entiende que las grandes y medianas empresas desde el punto de vista del capital, continúan existiendo e incluso tienden a aumentar, pero si se utiliza el parámetro del empleo se comprueba que éstas están disminuyendo ${ }^{11}$. En 1975, el tamaño medio de las empresas textiles en España era de 48'6 empleados y en la Comunidad de $27^{\prime} 5$.

Superada la crisis en 1985 , se comprueba que el porcentaje de pequeñas empresas ${ }^{12}$ continúa siendo elevado o incluso que ha aumentado. Los datos referidos a la Comunidad Valenciana muestran un predominio de la pequeña empresa (79\%), el sostenimiento de las grandes $(3 \%)$ y reducción de las medianas (Cuadro 2).

10 DALMAU PORTA, Juan Ignacio; DE MIGUEL FERNÁNDEZ, Enrique; MIQUEL PERIS, Salvador: Análisis estratégico de los sectores industriales y del turismo en la Comunidad Valenciana, 2 Volúmenes, Fundación de Estudios Bursátiles y Financieros, Universidad Politécnica de Valencia, Valencia, 1993.

11 Este hecho ofrece una repercusión indirecta sobre los parámetros de observación, pues si se toma el empleo como una unidad de referencia, se puede presentar la paradoja de que una gran empresa por el volumen de producción, de inversión de capitales etc. y que antaño contrataba 200 trabajadores, ahora con la innovación y modernización sólo ocupe a 25 o menos trabajadores y por tanto sea clasificada como mediana o pequeña.

12 Para 1985 y 1990 se consideran pequeñas empresas las comprendidas entre 1-19 trabajadores. 


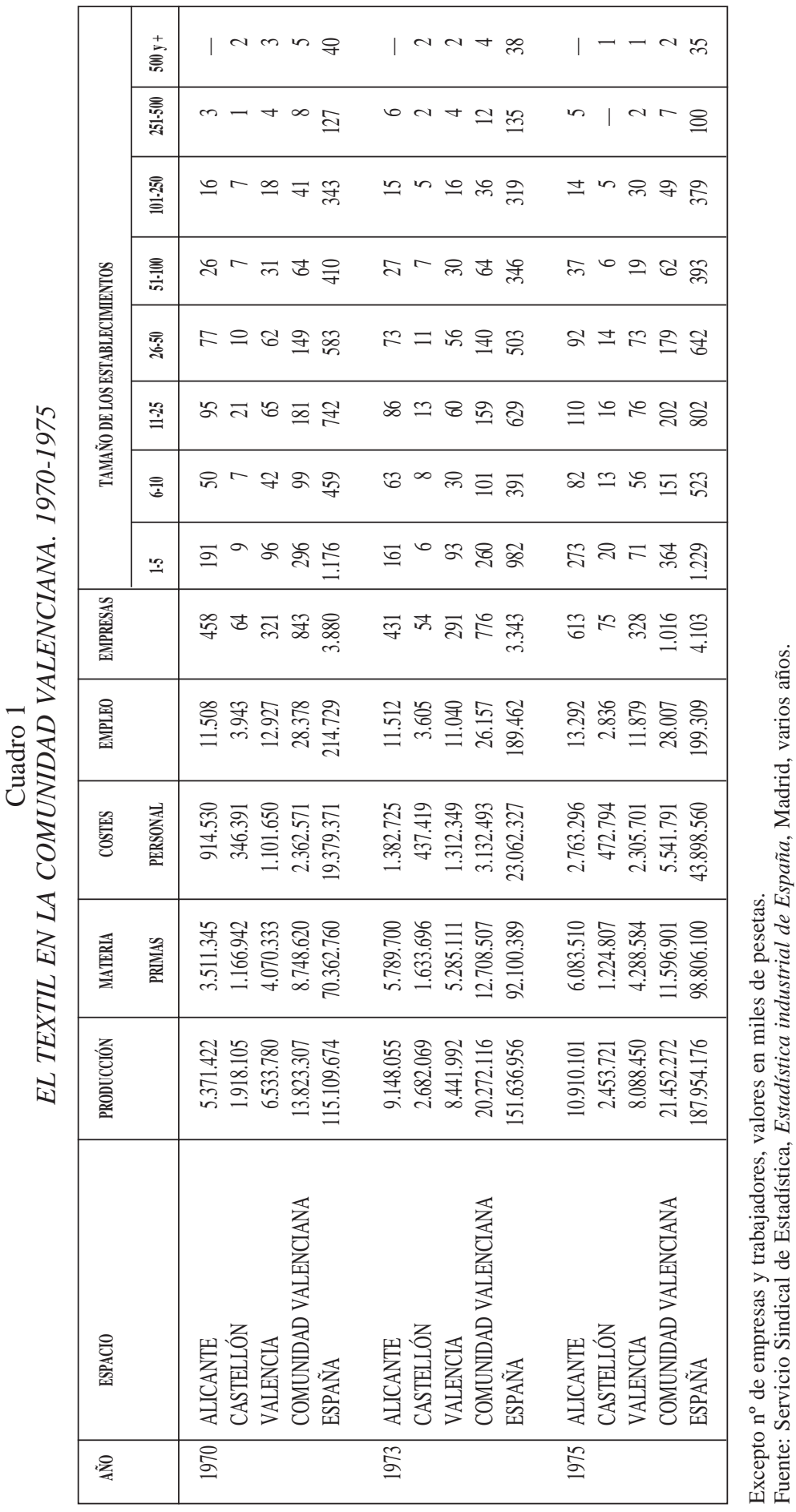




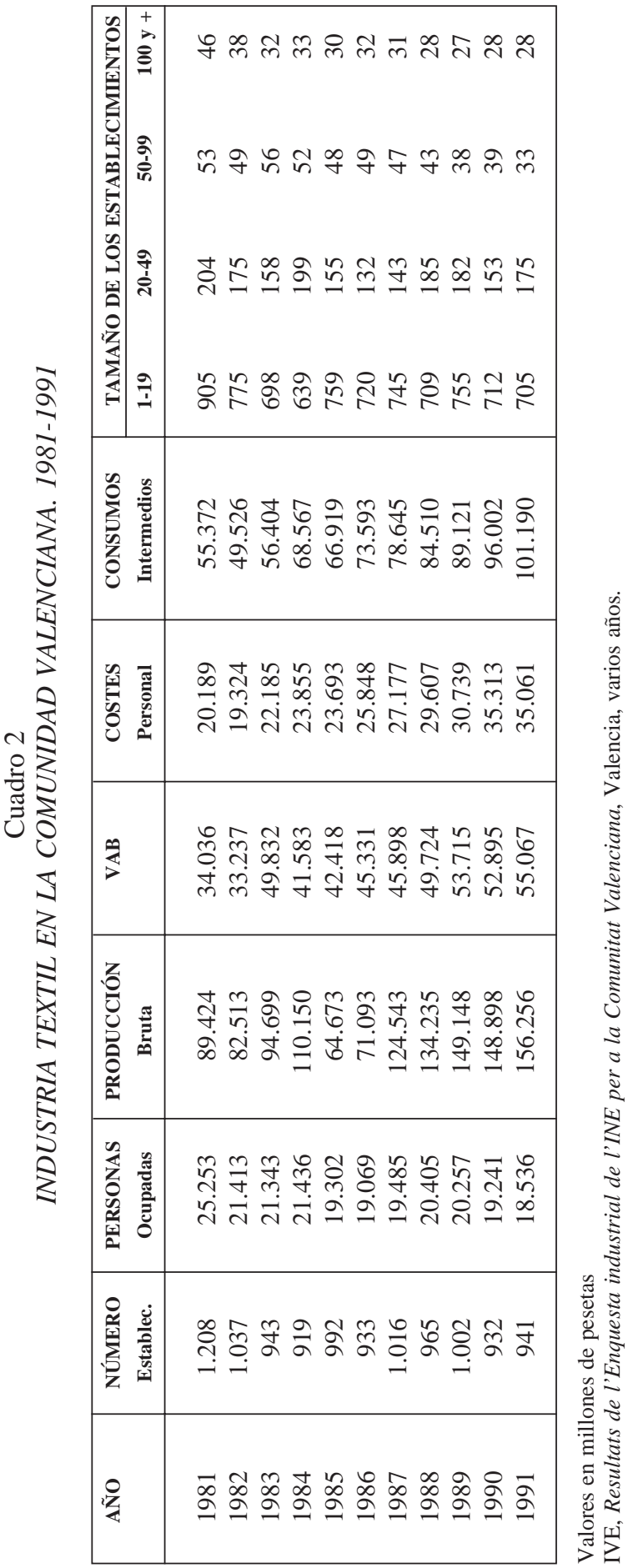


A finales del período, en 1991, la situación de la industria textil en cuanto al tamaño de las empresas, en la Comunidad apenas se había modificado, el 75\% eran pequeñas y el 3\% grandes. Además, no existía ninguna empresa en la provincia, según el directorio de empresas innovadoras, que superase los 500 trabajadores.

En la CEE, el sector textil mostró importantes cambios estructurales, pues a pesar del alto grado de fragmentación, la industria registró un considerable número de fusiones y adquisiciones ${ }^{13}$.

\section{b) La mano de obra, producción y costes}

La evolución del empleo, como se puede intuir de lo expuesto, no se puede definir como positiva. Los datos de la Encuesta Industrial indican un estancamiento o reducción de la mano de obra, tanto en las áreas geográficas estudiadas como en los distintos subsectores.

Entre 1970 y 1978 el empleo en el sector textil creció en la Comunidad Valenciana. A partir de 1978 y hasta 1991, la evolución fue negativa. La información procedente del INE así lo indica para la industria textil y de la confección. Del mismo modo los datos del Instituto Valenciano de Estadística confirman la crisis laboral en la actividad textil entre 1981 y 1991. La reducción del empleo no sólo se debió al cierre de empresas —el empleo descendió desde 1981 a 1991 el $27 \%$ y el número de establecimientos un $22 \%$-, sino también a otros factores como la mecanización de los procesos productivos.

Por consiguiente, la conclusión sobre la evolución del empleo y su impacto territorial consiste en que han sido las zonas tradicionales, centradas principalmente en el norte de la provincia y en los alrededores de Crevillent, las que más puestos de trabajo han perdido.

La costes de la mano de obra resultan de gran trascendencia dentro del conjunto de la producción y varían según los subsectores. De ellos dependen en gran medida la productividad y competitividad de esta industria, y en parte explican la inversión realizada en ampliación y modernización de la infraestructura de los procesos productivos. Estos gastos oscilan entre el $25 \%$ y $36 \%$ del total de los realizados.

La producción textil valenciana, pese a lo anteriormente expuesto, ha seguido una evolución ascendente, como indican los valores en pesetas efectivas. Sin embargo, en un estudio de los ciclos, en pesetas constantes, la evolución seguida por la producción textil, desde 1981, resulta negativa. El período más duro se sitúa entre 1981 y 1985; desde esta fecha hasta 1991 se produce una ligera recuperación. Se observa, por consiguiente, un impacto negativo previo, sin depender de una explicación monocausal, relacionado con la incorporación a la CEE y a la creación del Mercado Único. Sin embargo, una vez dentro de la CEE, se asiste a una recuperación de los niveles de producción.

Resulta obvio que el incremento de la productividad y la disminución del empleo se deben a la desaparición de las empresas marginales y a la modernización de los procesos productivos.

La materia prima empleada por el sector, desde 1970, era esencialmente de origen químico, puntualmente la lana y el algodón tenían una cierta importancia: es el caso de la lana en la industria de la alfombra y el algodón en las hilaturas. La producción de fibras químicas se hallaba dominada por la industria europea, y en concreto, la de poliester por Alemania (Hoechst) y la de Nylon por Gran Bretaña (ICI). La fabricación del hilado de algodón, estaba controlada por Alemania.

13 Comisión de las Comunidades Europeas, Panorama de la Industria Comunitaria, Servicio de Estadística Industrial de las Comunidades Europeas, Bruselas, 1991, capítulo 16, página 8. 
En general, el precio de las materias primas ha sido elevado, por lo que el margen de competitividad se ha reducido. Sobre el total de gastos, las materias primas constituyen uno de los capítulos más importantes a la par de la mano de obra, siendo en algún subsector incluso más elevada que ésta. En concreto, en el subsector de la hilatura, el precio de la materia prima puede suponer hasta el 50\% del valor final del producto.

Los costes de capital también han sido importantes, dada la carencia de recursos del sector y la dependencia del crédito; los costes de infraestructura han resultado onerosos, como en algún caso han puesto de manifiesto entrevistas con industriales o incluso el mismo presidente de la patronal textil.

\section{c) La demanda}

El mercado nacional ha estado reservado, casi hasta 1986, a nuestra industria textil, debido a la política proteccionista aplicada al sector, pues en algún caso como el de la confección, el arancel llegó hasta el 50\% del valor del producto importado. Los tratados del GATT y el acuerdo Multifibras desde 1974 beneficiaron a la industria española del sector, pues limitaron la importación desde los países que podían competir por el precio de la mano de obra, es decir, desde los que estaban en vías de desarrollo. Los acuerdos de cooperación con la Comunidad Económica Europea de 1978 tuvieron un impacto muy limitado. En 1981, en España se consumía el 57\% de lo fabricado por esta industria, pero desde mediados de la década de los 80 , con la integración con la CEE, se ha abierto nuestro mercado con el resultado de que las importaciones entre 1984 y 1990 en la Comunidad Valenciana se multiplicaron por 3'5 (en Alicante 3'1). Este incremento no se puede asociar a un crecimiento de la demanda de trapos y otros productos utilizados por los subsectores de hilaturas y regenerados, ya que la producción textil se hallaba estancada cuando no retrocedía.

Una constante tradicional de la industria textil ha sido su carácter exportador. La exportación ha evolucionado más en relación con la política monetaria y con el valor de la peseta en los mercados internacionales, que por aplicación de políticas exportadoras concretas. Una devaluación de la peseta permitió aumentar las exportaciones en 1985, una revalorización como la de finales de los ochenta la frenó. Sin embargo, visto de forma global, desde el ingreso en la CEE, la solidez del Sistema Monetario Europeo ha disminuido la capacidad competitiva de los productores europeos.

Los mercados exteriores principales han sido los de la CEE, los Países Árabes y los EEUU, siendo poco amplia la lista de los clientes. La demanda depende del tipo de producto textil.

La industria textil, durante este período, se abrió al comercio exterior y evolucionó diversificando sus mercados. La demanda procedente de la CEE, que en 1973 absorbía dos tercios de la exportación, se redujo hasta el $45^{\prime} 7 \%$, siendo ampliado el mercado con los países de Oriente Próximo.

La exportación textil también se caracteriza, de una forma global, por los bajos precios de los productos y poca calidad. De esta forma, la exportación española no puede hacer frente al diseño ni a la calidad que ofrecen los países más industrializados como Italia, ni a la innovación, tecnología y productividad de Alemania, Bélgica, Gran Bretaña o EEUU. Tampoco puede competir frente a los reducidos precios que ofrecen otros países en vías de industrialización, que cuentan con materia prima y/o con salarios irrisorios, como pueden ser, entre otros, desde China, Brasil, Marruecos o Turquía. Estos dos últimos países, además cuentan con acuerdos preferenciales con la CEE, lo que todavía contribuye a explicar mejor la crisis de las exportaciones. 
Los problemas de comercialización son importantes, estando los canales de distribución montados sobre esquemas antiguos. El tamaño reducido de las empresas y la competencia desleal (economía sumergida, copia de diseño, catálogos) ${ }^{14}$, contribuyen a explicar esta situación.

El sector textil fue aumentando su cuota de participación en las exportaciones totales de la Comunidad entre 1973 y 1982, consiguiendo el 11\% del total de lo vendido. Desde ese momento, se inicia un declive que dura hasta 1991, fecha en la que sólo aporta el 4'85\%. El sector en la Comunidad Valenciana, con una clara dependencia del mercado europeo, registró una tendencia positiva hasta 1984, año en el que alcanzó su máximo, desde este momento ha retrocedido y en 1991 todavía no se había igualado el máximo anterior. Las exportaciones valencianas, además, han ido perdiendo importancia en el marco del Estado, pues mientras en 1985 les correspondía el 29'5\% de las exportaciones textiles, en 1991 se sitúan en el 20`4\%. La exportación textil alicantina, por su parte, también ha mostrado esta tendencia regresiva, ya que el volumen de lo exportado ha descendido incluso en pesetas corrientes, aunque no de una forma tan intensa, y su aportación al total de la Comunidad entre 1989 y 1991 ha pasado de un 34\% a un 31\%.

El mercado interior de los años setenta había perdido ya su característica de postguerra, es decir, de bajo poder adquisitivo y consumidor de productos de baja calidad. El crecimiento demográfico de los años sesenta y el aumento de la capacidad de compra de los españoles, repercutió en un incremento de la demanda del sector. Esta situación benefició a la industria textil hasta mediados de los ochenta; sin embargo, la apertura del mercado interior, como señalan las estadísticas, significó una pérdida progresiva de éste. De este modo, en 1991 el valor de las importaciones casi había igualado al de las exportaciones, aunque la tasa de cobertura seguía siendo positiva para el sector.

\section{Inversión}

\section{a) Evolución de la inversión}

La inversión textil, dentro de la Comunidad Valenciana, ha presentado una evolución positiva (pts. corrientes) durante el período 1973-1991, pero en pesetas constantes ha registrado un espectacular retroceso. La inversión recogió su peor momento durante 198083. Posteriormente se produjo una recuperación con grandes oscilaciones, que evidenció una incertidumbre en el sector. El aumento (menor en pts. constantes), realmente encubre una debilidad estructural muy importante, ya que si se compara esta variable sectorial con el conjunto de la inversión industrial queda manifiesta su escasa aportación al total. En este sentido, cabe destacar que las inversiones textiles en 1973 fueron el 17\% del total de las industriales y en 1991 sólo el 2’7\% (cuadro 3).

El punto de partida era positivo a comienzos de los setenta; existía un gran optimismo sobre las posibilidades de invertir y crear una empresa. El factor psicológico, por tanto, era un elemento a tener en cuenta. En declaraciones de la época se afirmaba: «hoy día es relativamente fácil ser industrial. Sólo basta con tener unos cuantos telares...» o «El sol sale para todos. Y las oportunidades que yo he tenido las han podido disfrutar todos».

En 1975, el sector textil se hallaba en plena reestructuración. La crisis de finales de la década de los ochenta destaca el papel de los polos hegemónicos, por ser el reducto donde

14 DUARTE, Agustín: «Análisis de los subsectores industriales representativos», Estructura económica de la provincia de Alicante, Diputación provincial de Alicante, 1993, pp. 189-208. 


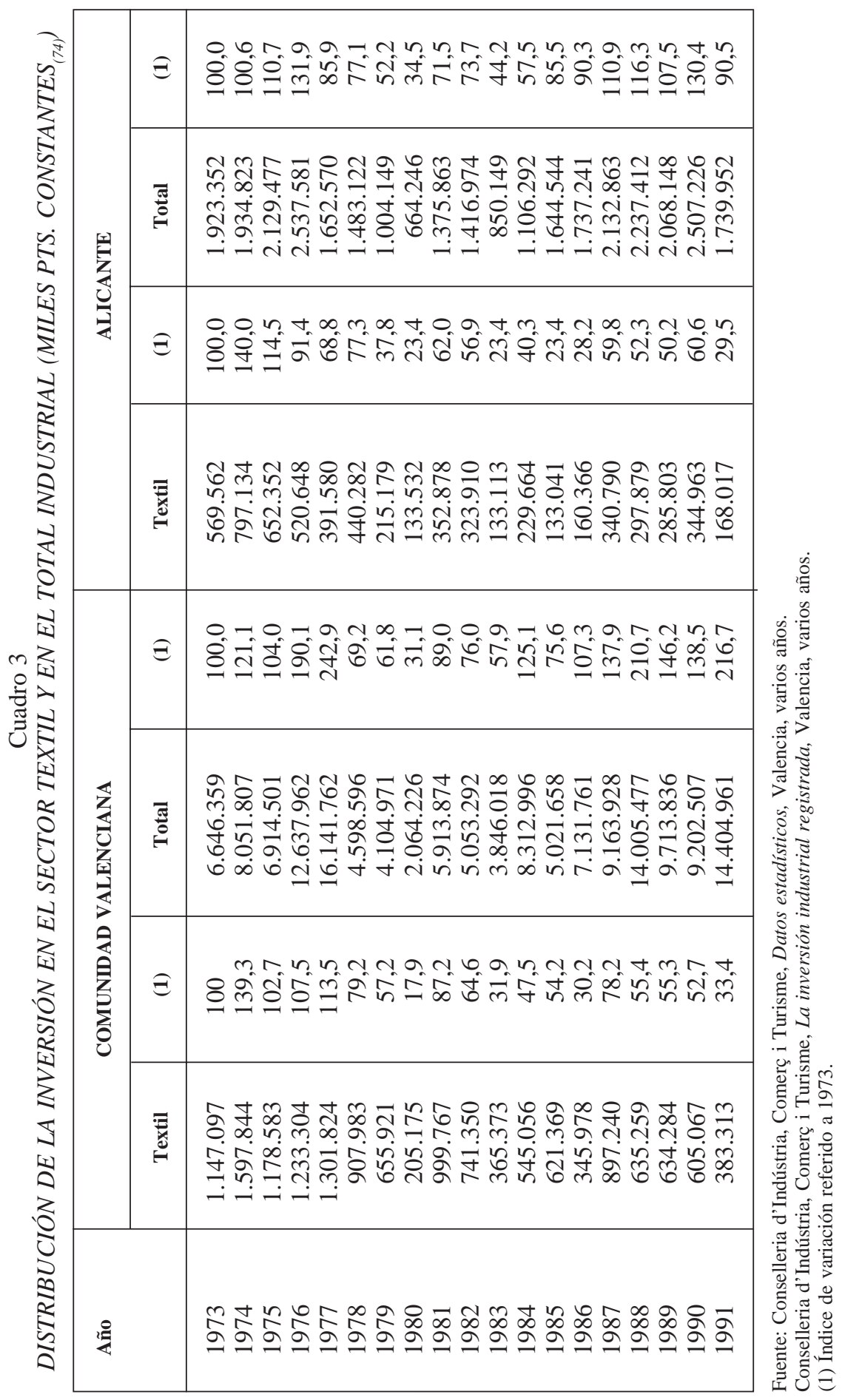


se continúan realizando las inversiones, y reduce el hinterland de los polos a los municipios donde la actividad textil se ha consolidado.

La expansión económica de 1985 se reconoce al observar un mayor número de municipios donde se invierte; la comarca septentrional aparece con el centro difuminado por la intensidad de las inversiones en los municipios vecinos. Este hecho se debió a una saturación y congestión del polo (Alcoi), que carecía de una buena infraestructura y padecía una mayor presión fiscal, lo que repercutió en una fuga hacia los municipios colindantes.

\section{b) Factores de los que depende la inversión}

La inversión depende de una gran cantidad de variables, muchas de las cuales resultan de difícil cuantificación. No obstante, se han seleccionado algunas de ellas por lo significativas que pueden resultar en la explicación de un hecho de gran trascendencia, la repercusión en la distribución y organización espacial.

El primero de ellos, siguiendo el orden marcado en la exposición, corresponde al tamaño de las empresas. Es lógico que el reducido tamaño de éstas indique un predominio de la empresa familiar, muchas veces de origen artesanal, cuyo gerente es el mismo dueño y que normalmente acude al crédito privado y a la autofinanciación para conseguir el capital necesario para invertir. Estas empresas habitualmente tienen dificultades para estar informadas sobre las políticas financieras públicas y, cuando les llega la información, la burocracia supone una dura barrera ${ }^{15}$. Además, en ocasiones las ayudas prometidas han sido cuestión de polémica ${ }^{16}$. La necesidad de conseguir cada vez mayores sumas de dinero para comprar maquinaria, o mejorar la infraestructura, etc., dificulta tanto la modernización de la empresa como el acceso a los mercados. La reconversión industrial iniciada en los últimos años de la década de los sesenta e inicios de los setenta, ha contribuido a la concentración empresarial, quedando marginadas muchas empresas, no por la falta de habilidad de sus trabajadores o empresarios, sino por la carencia del capital necesario para modernizar su equipo o estructura productiva.

La llegada de capital hacia la industria textil se ha visto dificultada por la penosa marcha del sector, como demuestran algunas variables (exportación, producción). El cierre de empresas condujo inicialmente a la economía subterránea y definitivamente a la extinción; en algún caso se produjo un traslado de las instalaciones productivas a países donde la mano de obra era más barata. El resultado final ha sido siempre el mismo: crisis y paro en unas comarcas y municipios donde la economía dependía casi exclusivamente de este sector. Las inversiones, por tanto, sólo pudieron ser atractivas en casos específicos de empresas altamente innovadoras, que incorporaran nuevas tecnologías y equipos y que dispusieran de mercados exteriores consolidados o con expectativas positivas.

A las dificultades que atraviesa la pequeña empresa para acceder a los capitales, se suman otros elementos que, para ella y debido a su estructura, le resultan grandes obstáculos: la recesión de la demanda unida a una carencia de reservas, la necesidad de formación

15 En esta dirección han coincidido numerosas publicaciones.

16 Informe Peat Marcwich, volumen 13 «Sin embargo se ha producido una proliferación de ayudas al sector de distintos tipos, entidades y administraciones creando expectativas, que luego no se han cumplido dada la regulación comunitaria a este respecto, y que perjudican a la credibilidad de la Administración. Las ayudas a las inversiones en activos fijos están prácticamente vetadas, por lo que las políticas de apoyo deberán dirigirse a otras medidas como son el apoyo a las PYMES en la mejora de la calidad, diseño, innovación tecnológica, mejora de la gestión y potenciación de la comercialización». 


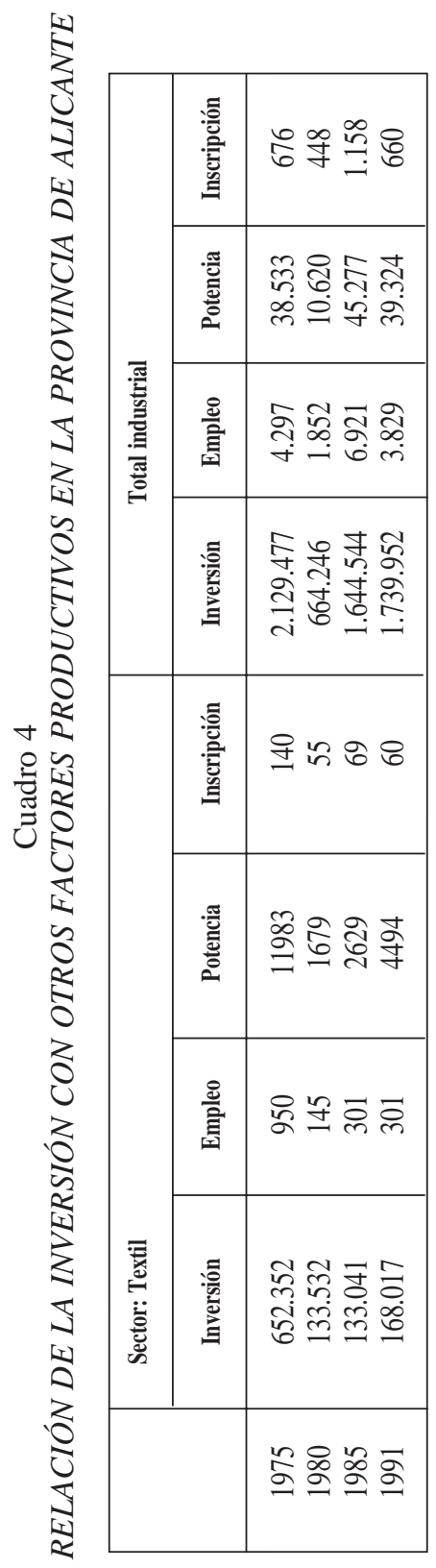

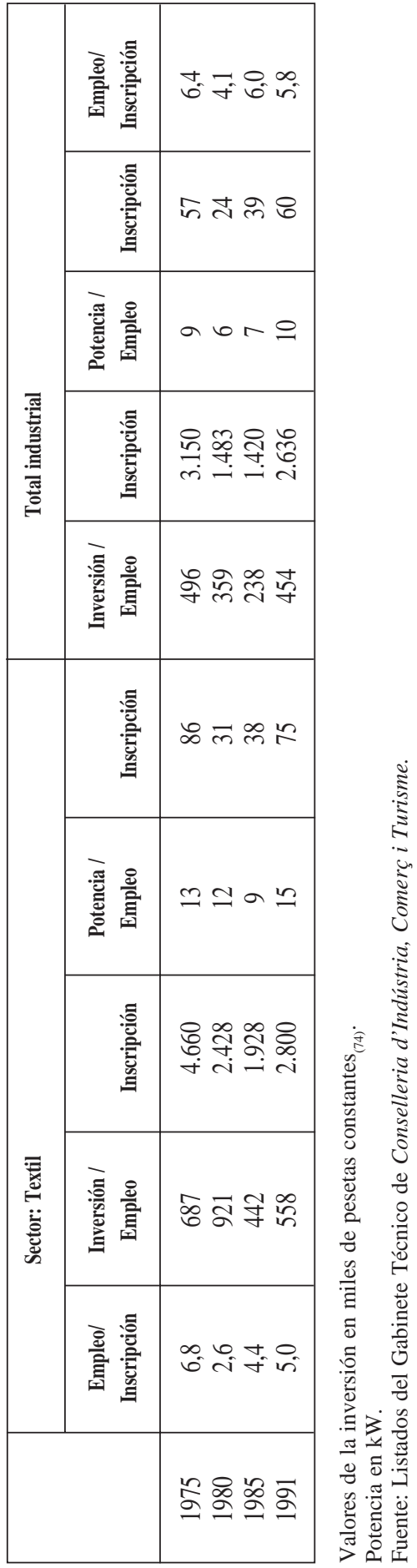


tanto de empresarios como de trabajadores, la ausencia de una adecuada infraestructura, la política fiscal, etc. ${ }^{17}$

La relación entre la inversión y el empleo ha sido inversamente proporcional, pues aunque en apariencia la inversión ha aumentado, realmente no ha sucedido así (pts. constantes), y los dos factores se han visto involucrados en una profunda crisis, siendo mayor en el segundo. Según la información disponible (Cuadro 4), el capital necesario para crear un puesto de trabajo, aunque se ha reducido entre 1975 y 1991, siempre ha sido mayor a la media de las industrias alicantinas. Por consiguiente, si la demanda de capital es mayor que en los otros sectores industriales, las rentabilidades comparadas frenan la inversión, y si la inversión no crece, no se crea empleo; en segundo lugar, si lo que se busca es un aumento de la competitividad, los costes salariales constituyen un freno importante a este proceso, ya que crecen pese a la reducción de los empleos. Queda claro que el destino de la inversión se dirige a reducir estos costes e indirectamente el empleo. La inversión/inscripción presenta casi las mismas características que las del empleo: una inversión superior a la media de las industrias alicantinas.

Otra posibilidad es reducir los costes de energía y materias primas; sin embargo como la mayor parte son importados, pagados en divisas, y con frecuencia sus precios controlados por multinacionales, la acción empresarial en busca de alternativas, tanto en la diversificación de consumos como en la innovación tecnológica, que permitan ahorrar en los inputs, encuentra pocas opciones.

Respecto a la potencia contratada, ésta ha aumentado en relación a los puestos de trabajo previstos crear, con lo que se evidencia la modernización del equipo productivo. La potencia/inscripción ha aumentado desde 1980 a 1991, como consecuencia de la renovación del equipo productivo. No obstante, en conjunto la tendencia manifiesta por las industrias europeas y, en concreto, por las del sector, es la de mejorar los aprovechamientos energéticos, por lo que se espera que finalmente este último aspecto contribuya a un descenso de la potencia instalada.

El valor de la producción, como ya se ha expuesto, aparentemente ha evolucionado de forma favorable durante todo el período, sin embargo y pese a la recuperación de 1988, se comprueba que desde 1980 es recesiva (pesetas constantes). De esta forma, el estímulo del beneficio no es positivo y los excedentes de capital, que en esta industria han permitido la autofinanciación, siguen la misma tendencia. La inversión pasó en 1978 de ser el 1'6\% de la producción y el 3'9\% del VAB, al 0'6\% y 2\% respectivamente en 1991.

En la CEE por el contrario, la inversión ha crecido más que la producción (índices de variación). Una de las consecuencias ha sido la reducción del empleo y por tanto, el incremento de la productividad, siendo ésta y la inversión los que mayor crecimiento han registrado en los índices de variación.

Las conclusiones que se desprenden de lo expuesto son: en primer lugar, que la evolución de la producción y las exportaciones no favorecen ni estimulan la inversión, y en segundo lugar que a las empresas se les va reduciendo el excedente de capital.

La cantidad de dinero necesaria para realizar una inversión se fue comparativamente elevando, entre otras razones por la mayor complejidad de las máquinas y por las normativas que exigían mejoras en sanidad, seguridad y tratamiento de residuos. En este sentido,

17 CARDONA, Ricardo: «El textil valenciano: sus características y perspectivas», Información Comercial Española, n ${ }^{\circ}$ 586, Madrid, Junio 1982, pp. 125-127.

«La disminución generalizada de los beneficios, las mayores cargas fiscales y la falta de perspectivas económicas, ha frenado la inversión, convirtiendo en más obsoleto un parque de maquinaria que se encontraba a la altura de las necesidades. (...)». 
la distribución en porcentajes de la inversión señala un aumento progresivo de los gastos en infraestructura y otros, mientras que el correspondiente a maquinaria se fue reduciendo. La deficiencia en infraestructura y dotación pública de espacios industriales acondicionados sobresale en este sector, ya que la tendencia en este capítulo es ascendente, y se debe relacionar con las deficiencias, en concreto, de algunas ciudades como Alcoi. En 1975 el 7'5\% de la inversión registrada se dedicó a infraestructura y «otros», en 1991 fue del $20{ }^{\prime} 4 \%$.

Si la inversión en el sector textil se compara con la media industrial de la provincia, se puede concluir que la inversión por inscripción resultó más cuantiosa y generó menos empleo. Por tanto, el desvío de los capitales hacia otras actividades, donde con menos dinero y riesgo se puedan obtener los mismos o más beneficios, es una opción a tener en cuenta en la evolución futura de esta industria y en una diversificación del tejido industrial en la provincia de Alicante.

La inversión textil en ampliación ha sido mayoritaria durante todo el período. El predominio de la ampliación se vincula al carácter de autofinanciación de la industria textil. Queda pues de manifiesto el carácter intensivo en el uso del capital que ha ido adquiriendo la industria textil para modernizar las empresas y conseguir con ello reducir los costes laborales, y para compensar el aumento de otros gastos con la finalidad de resultar más competitiva.

\section{c) Distribución geográfica}

La inversión textil en la Comunidad Valenciana se ha concentrado en su mayor parte en las provincias de Alicante y de Valencia, alternando las dos provincias en la primera posición. La industria textil en la provincia de Alicante se encuentra en una fase de crisis, como demuestra que del total de las inversiones industriales, las textiles en 1975 representaban el 29'5\% y en 1991 sólo el 6'5\%. El mayor beneficio obtenido por las inversiones en otras actividades económicas explica esta recesión. El sector calzado está desplazando a la actividad textil en bastantes municipios. El caso de Crevillent es significativo; un municipio que en 1975 concentraba el 92'73\% de su inversión en el textil, en 1991 se hallaba próximo a ser sustituido por el calzado como sector hegemónico, y el textil tan sólo atraía el 38'76 \% de la inversión. Otros municipios, como Callosa de Segura, Catral o Villena, se encontraban en una situación similar. El sector del juguete también ha desplazado al textil en algunas poblaciones como es el caso de Biar.

La condición de input para otros sectores industriales, como los dos mencionados anteriormente, unido al mercado que genera el turismo o las grandes urbes, explican la difusión de la inversión textil en municipios como Altea, Alacant, Benidorm y Elx.

No obstante, la estructura industrial profunda no se ha modificado: Crevillent y Alcoi continúan siendo importantes núcleos polarizadores de la actividad textil. En el Norte de la provincia, las alternativas para la sustitución no han sido tan óptimas, ya que las tradicionales industrias papeleras y metalúrgicas se hallan en crisis. En algún momento y en determinados municipios, la industria de la alimentación, juguetes o los servicios, se han presentado como alternativas, pero sin desplazar en su protagonismo al sector textil.

La inversión textil provincial se ha concentrado alrededor de Alcoi (19\% en 1975, 50\% en $1980,17 \%$ en 1985 y $22 \%$ en 1991). Otros municipios próximos, igualmente recogieron una parte importante de la inversión: Banyeres (9\% en1975 y el 16\% en 1985), Cocentaina (el $16 \%$ en 1980 y el $10 \%$ en 1985) y Muro (12\% en 1975, 10\% en 1980). Pero el textil también ha sido predominante o hegemónica en municipios próximos como Alcosser, Beneixama, Beniarrés, Benilloba, Benimarfull, Gorga, L'Orxa y otros no tan próximos 


\section{Cuadro 5}

LOCALIZACIÓN DE LA INVERSIÓN EN LA PROVINCIA DE ALICANTE Industria textil $(1975,1980,1985$ y 1991)

\begin{tabular}{|c|c|c|c|c|c|c|c|c|c|c|}
\hline & \multicolumn{5}{|c|}{1975} & \multicolumn{5}{|c|}{1980} \\
\hline Municipio & total & textil & (1) & (2) & (3) & total & textil & (1) & (2) & (3) \\
\hline \multicolumn{11}{|l|}{ Alacant } \\
\hline \multicolumn{11}{|l|}{ Albatera } \\
\hline Alcoi & 185.338 & 137.275 & 74,1 & Textil & 19,29 & 237.448 & 156.733 & 66,01 & Textil & 50,48 \\
\hline Alcosser & 9.970 & 9.970 & 100,0 & Textil & 1,40 & & & & & \\
\hline \multicolumn{11}{|l|}{ Almoradí } \\
\hline Alqueria d'Aznar & 179.277 & 1.837 & 1,0 & Papel & 0,26 & & & & & \\
\hline \multicolumn{11}{|l|}{ Altea } \\
\hline Aspe & 29.268 & 2.329 & 8,0 & Caucho y plástico & 0,33 & & & & & \\
\hline Banyeres & 86.240 & 65.226 & 75,6 & Textil & 9,16 & 21.158 & 6.844 & 32,35 & Textil & 2,20 \\
\hline \multicolumn{11}{|l|}{ Beneixama } \\
\hline \multicolumn{11}{|l|}{ Beniarrés } \\
\hline \multicolumn{11}{|l|}{ Benidorm } \\
\hline Benilloba & 10.070 & 8.940 & 88,8 & Textil & 1,26 & & & & & \\
\hline Benimarfull & 666 & 666 & 100,0 & Textil & 0,09 & & & & & \\
\hline Biar & 17.369 & 105 & 0,6 & Juguete & 0,01 & & & & & \\
\hline Callosa Segura & 14.297 & 9.708 & 67,9 & Textil & 1,36 & 21.093 & 5.805 & 27,52 & Caucho y plástico & 1,87 \\
\hline Camp de Mirra & 332 & 250 & 75,3 & Textil & 0,04 & & & & & \\
\hline \multicolumn{11}{|l|}{ Catral } \\
\hline Cocentaina & 40.375 & 37.415 & 92,7 & Textil & 5,26 & 56.932 & 52.232 & 91,74 & Textil & 16,82 \\
\hline Cox & 23.812 & 12.570 & 52,8 & Textil & 1,77 & 3.355 & 3.355 & 100,00 & Textil & 1,08 \\
\hline Crevillent & 97.865 & 90.748 & 92,7 & Textil & 12,75 & 58.468 & 37.050 & 63,37 & Textil & 11,93 \\
\hline Elx & 234.202 & 48.338 & 20,6 & Calzado & 6,79 & & & & & \\
\hline Gata de Gorgos & 9.082 & 94 & 1,0 & Cuero & 0,01 & & & & & \\
\hline \multicolumn{11}{|l|}{ Gorga } \\
\hline L'Orxa & 4.523 & 4.473 & 98,9 & Textil & 0,63 & & & & & \\
\hline Muro & 94.169 & 90.726 & 96,3 & Textil & 12,75 & 39.889 & 31.750 & 79,60 & Textil & 10,23 \\
\hline Mutxamel & 127.250 & 125.345 & 98,5 & Textil & 17,61 & & & & & \\
\hline Orihuela & 38.982 & 52 & 0,1 & Construcción & 0,01 & & & & & \\
\hline \multicolumn{11}{|l|}{ Orxeta } \\
\hline Pego & 7.571 & 55 & 0,7 & Bebida y tabaco & 0,01 & 30.147 & 16.692 & 55,37 & Textil & 5,38 \\
\hline \multicolumn{11}{|l|}{ Rafal } \\
\hline \multicolumn{11}{|l|}{ Redován } \\
\hline \multicolumn{11}{|l|}{ Sant Vicent } \\
\hline \multicolumn{11}{|l|}{$\operatorname{Sax}$} \\
\hline \multicolumn{11}{|l|}{ Torrevieja } \\
\hline Villena & 83.193 & 65.594 & 78,8 & Textil & 9,22 & & & & & \\
\hline Total & 2.441 .438 & 711.716 & 29,2 & & & 1.702 .888 & 310.461 & 18,23 & & \\
\hline
\end{tabular}

* Inversión en miles de pesetas. 
Cuadro 5 (continuación)

LOCALIZACIÓN DE LA INVERSIÓN EN LA PROVINCIA DE ALICANTE Industria textil $(1975,1980,1985$ y 1991)

\begin{tabular}{|c|c|c|c|c|c|c|c|c|c|c|}
\hline & \multicolumn{5}{|c|}{1985} & \multicolumn{5}{|c|}{1991} \\
\hline Municipio & total & textil & (1) & (2) & (3) & total & textil & (1) & (2) & (3) \\
\hline Alacant & & & & & & 2.573 .833 & 2.268 & 0,09 & Construcción & 0,29 \\
\hline Albatera & 39.094 & 855 & 2,19 & Confección & 0,15 & 137.603 & 51.076 & 37,12 & Textil & 6,43 \\
\hline Alcoi & 311.205 & 95.156 & 30,58 & Textil & 17,13 & 467.756 & 173.769 & 37,15 & Textil & 21,87 \\
\hline Alcosser & & & & & & 8.530 & 8.530 & 100,00 & Textil & 1,07 \\
\hline Almoradí & 59.413 & 3.245 & 5,46 & Alimentación & 0,58 & & & & & \\
\hline Alqueria d'Aznar & & & & & & 49.778 & 49.778 & 100,00 & Textil & 6,26 \\
\hline Altea & 33.306 & 70 & 0,21 & Fabricación productos metal & 0,01 & & & & & \\
\hline \multicolumn{11}{|l|}{ Aspe } \\
\hline Banyeres & 107.265 & 89.720 & 83,64 & Textil & 16,15 & 295.141 & 121.970 & 41,33 & Textil & 15,35 \\
\hline Beneixama & 20.643 & 14.500 & 70,24 & Textil & 2,61 & 79.905 & 73.475 & 91,95 & Textil & 9,25 \\
\hline Beniarrés & 1.018 & 600 & 58,94 & Textil & 0,11 & & & & & \\
\hline Benidorm & 106.892 & 2.393 & 2,24 & Construcción & 0,43 & & & & & \\
\hline \multicolumn{11}{|l|}{ Benilloba } \\
\hline \multicolumn{11}{|l|}{ Benimarfull } \\
\hline Biar & 45.884 & 13.000 & 28,33 & Confección & 2,34 & & & & & \\
\hline Callosa Segura & 25.598 & 7.300 & 28,52 & Calzado & 1,31 & & & & & \\
\hline Camp de Mirra & 3.750 & 2.520 & 67,20 & Textil & 0,45 & & & & & \\
\hline Catral & 76.418 & 51.440 & 67,31 & Textil & 9,26 & 122.089 & 11.310 & 9,26 & Calzado & 1,42 \\
\hline Cocentaina & 74.235 & 58.088 & 78,25 & Textil & 10,46 & 219.111 & 80.964 & 36,95 & Textil & 10,19 \\
\hline Cox & 30.657 & 16.483 & 53,77 & Textil & 2,97 & 135.982 & 15.075 & 11,09 & Fabricación productos metal & 1,90 \\
\hline Crevillent & 287.954 & 175.422 & 60,92 & Textil & 31,57 & 298.414 & 115.672 & 38,76 & Textil & 14,56 \\
\hline Elx & 901.218 & 1.020 & 0,11 & Calzado & 0,18 & 981.195 & 10.250 & 1,04 & Calzado & 1,29 \\
\hline \multicolumn{11}{|l|}{ Gata de Gorgos } \\
\hline Gorga & & & & & & 5.853 & 5.853 & 100,00 & Textil & 0,74 \\
\hline \multicolumn{11}{|l|}{ L'Orxa } \\
\hline Muro & 66.718 & 9.274 & 13,90 & Agua y energía & 1,67 & 117.747 & 44.425 & 37,73 & Textil & 5,59 \\
\hline \multicolumn{11}{|l|}{ Mutxamel } \\
\hline Orihuela & & & & & & 980.922 & 189 & 0,02 & Agua y energía & 0,02 \\
\hline Orxeta & 580 & 500 & 86,21 & Textil & 0,09 & & & & & \\
\hline \multicolumn{11}{|l|}{ Pego } \\
\hline Rafal & & & & & & 7.799 & 3.171 & 40,66 & Alimentación & 0,40 \\
\hline Redován & 79.269 & 7.275 & 9,18 & Otros servicios & 1,31 & 75.432 & 1.748 & 2,32 & Fabricación productos metal & 0,22 \\
\hline Sant Vicent & 246.900 & 2.462 & 1,00 & Alimentación & 0,44 & 416.820 & 22.300 & 5,35 & Caucho y plástico & 2,81 \\
\hline Sax & 64.114 & 4.258 & 6,64 & Calzado & 0,77 & & & & & \\
\hline Torrevieja & & & & & & 151.790 & 2.731 & 1,80 & Construcción & 0,34 \\
\hline \multicolumn{11}{|l|}{ Villena } \\
\hline Total & 8.039 .092 & 555.581 & 6,91 & & & 12.185 .750 & 794.554 & 6,52 & & \\
\hline
\end{tabular}

Elaborado a partir de los listados de la Conselleria d'Indústria, Comerç i Turisme.

(1) Sector*100/Total municipal.

(2) Sector predominante o hegemónico.

(3) Sector*100/Total sector provincial.

* Inversión en miles de pesetas. 


\section{Mapa 1}

Distribución de la inversión en la provincia de Alicante

Sector: Textil (1975, 1980, 1985, 1991)
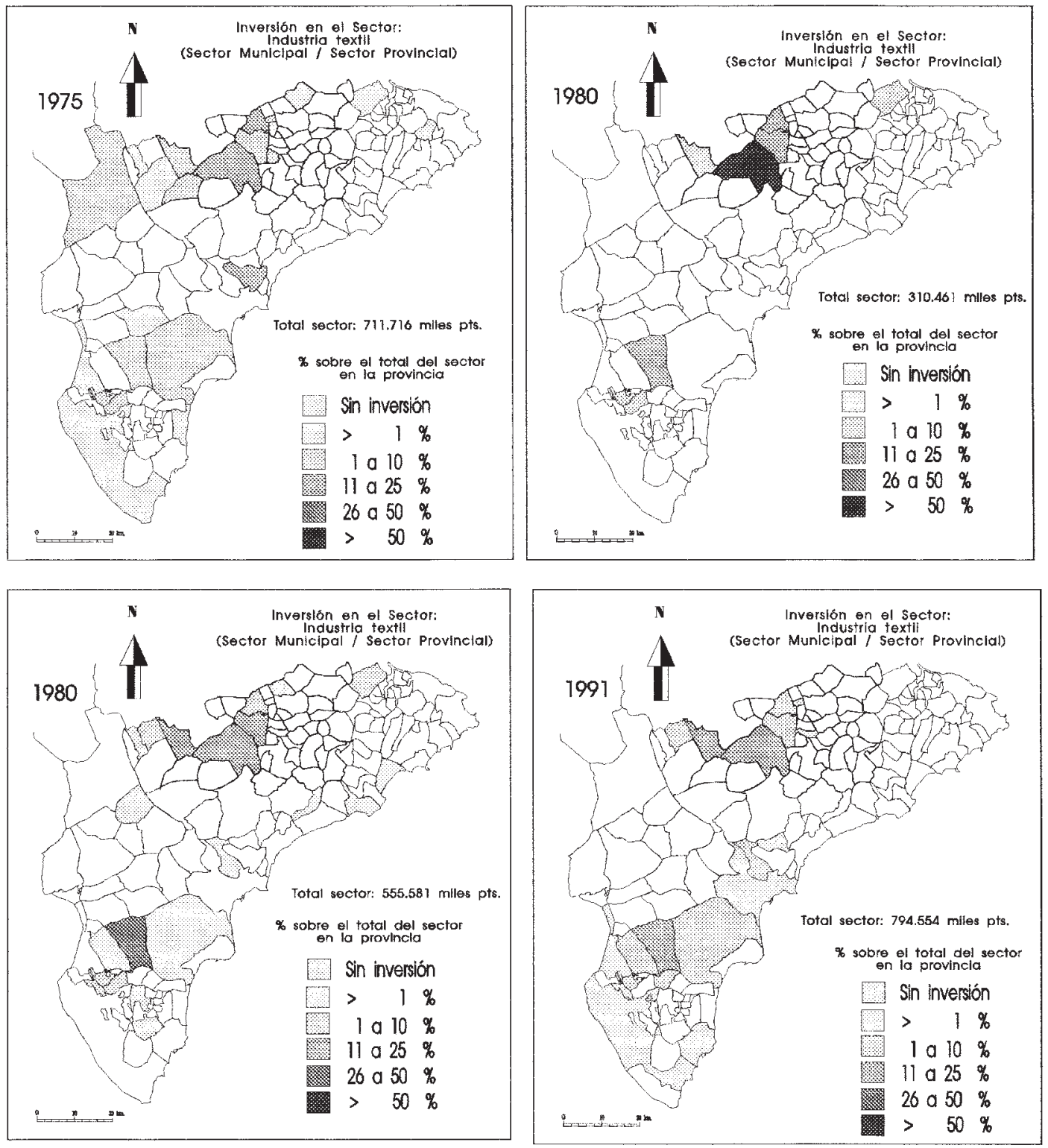
como Mutxamel o Villena. La saturación y deficiencia en la infraestructura industrial de Alcoi contribuyen a la difusión espacial de la industria textil, como en parte evidencian los anteriores datos. En Crevillent también se ha producido una importante concentración de la inversión textil provincial ( $12 \%$ en $1975,12 \%$ en $1980,31 \%$ en 1985 y $14 \%$ en 1991) que sitúan a la localidad como núcleo vertebrador del sector textil en el sur de la provincia de Alicante.

En la localización de la inversión textil se observan, pues, las siguientes características: 1.- Dos núcleos vertebradores: Alcoi y Crevillent.

2.- En los períodos de expansión económica, se asiste a una difusión espacial (movimientos centrífugos), con asentamientos en zonas tradicionalmente agrícolas.

3.- En los momentos de recesión, la inversión textil se vuelve a concentrar en los municipios con tradición en el sector (movimientos centrípetos). 
